\title{
Madres, padres y profesores como educadores de la resiliencia en niños colombianos
}

\author{
Victoria Eugenia Cabrera García \\ Universidad de La Sabana - Bogotá - Colombia \\ Viviana Lucía Aya Gómez \\ Universidad de La Sabana - Bogotá - Colombia \\ Diana Sthefanía Muñoz Gómez \\ Universidad de La Sabana - Bogotá - Colombia \\ Ivón Paola Guevara Marín \\ Universidad de La Sabana - Bogotá - Colombia \\ Andrés Mauricio Cano Rodas \\ Universidad de La Sabana - Bogotá - Colombia
}

\section{Resumen}

El objetivo del estudio consistió en analizar el aporte de las prácticas educativas de madres, padres y profesores en el desarrollo de la resiliencia en niños(as). Se contó con 417 niños(as), 199 profesores, 111 mamás y 80 papás de zona rural de nivel socioeconómico bajo. Fue un estudio transversal, de análisis correlacional, explicativo. Las niñas mostraron mayor nivel de generosidad, laboriosidad y resiliencia total en comparación con los niños. Las mamás tienden a practicar la aceptación y apoyo, la afirmación del poder, el retiro del afecto y el trato rudo más que los papás. Las profesoras también ejercen estás prácticas pero en menor proporción que los dos padres. La aceptación de papás y profesoras, el monitoreo de los dos padres y la afirmación del poder de las profesoras, son variables que pesan de manera significativa en la explicación de resiliencia en los niños(as). Es importante diseñar estrategias de intervención conjuntas en el contexto familiar y escolar desde edades tempranas, con el fin de desarrollar procesos de resiliencia en niños, teniendo en cuenta el papel protagónico de los padres y los profesores en este proceso.

Palabras clave: Educación; resiliencia; niños.

\section{Mothers, fathers and teachers as educators of resilience in Colombian children}

\begin{abstract}
The aim of this study was to analyze the contribution of the educational practices of parents and teachers in the development of resilience in children. The sample included 417 children, 199 teachers, 111 mothers and 80 fathers of a rural setting of low socioeconomic level. It was an explanatory, cross-sectional study, with a correlational analysis. The girls showed higher levels of generosity, diligence and overall resilience compared to boys. Mothers tended to practice acceptance and support, assertion of power, withdrawal of affection and harsh parenting more than fathers. Teachers also exercised these practices but to a lesser extent than two parents. Acceptance by parents and teachers, parental monitoring and teacher affirmation of power, were variables that weighed significantly in explaining resilience in children. It's important to design intervention strategies within the family and school's context from a young age, to help children develop resilience, taking into account the crucial role of parents and teachers in this process.
\end{abstract}

Keywords: Education; resilience; children.

\section{Mães, pais e professores como educadores da resiliência em crianças colombianas}

\section{Resumo}

O objetivo do estudo consistiu em analisar a influência das práticas educativas de mães, pais e professores no desenvolvimento da resiliência em crianças. Participaram 417 crianças, 199 professores, 111 mães e 80 pais de zona rural de baixo nível socioeconômico. Foi um estudo transversal, de análise correlacional, explicativa. As meninas mostraram maior nível de generosidade, dedicação ao trabalho e resiliência total em comparação aos meninos. As mães tendem a mais praticar a aceitação e o apoio, a afirmação do poder, a retirada do afeto e o tratamento rude do que os pais. As professoras também exercem essas práticas, mas em menor proporção do que os pais. A aceitação de pais e professoras, o monitoramento de pais e mães e a afirmação do poder das professoras são variáveis que pesam de forma significativa na explicação da resiliência nas crianças. É importante projetar estratégias de intervenção conjuntas no contexto familiar e escolar desde idades menores, a fim de desenvolver processos de resiliência em crianças, tendo em conta o papel protagonista dos pais e professores nesse processo.

Palavras-chave: Educação; resiliência; crianças. 


\section{Introducción}

La edad escolar resulta clave en la historia de niños y niñas (niños de aquí en adelante). Según Meresman (2005) es en la infancia, especialmente en la edad escolar, en donde se moldean las preferencias, costumbres y estilos de vida de cada persona. Por su parte Rutter (2006) señala que es importante identificar los factores de riesgo y protección en la infancia, porque permiten predecir resultados negativos o positivos en el proceso de un desarrollo saludable. De esta manera, tanto la vulnerabilidad como la protección se configuran como procesos interactivos que actúan simultáneamente en el proceso de desarrollo educativo.

En algunos países, concretamente en Colombia, se viven altos niveles de pobreza. Esta ha sido descrita como un factor de riesgo que genera dolor y estrés. Diversos autores la sitúan al mismo nivel de vivir con padres con alguna patología mental severa (Fonagy, Steele, Steele, Higgitt, \& Target, 1994). Las familias que viven en situación de pobreza están expuestas, con frecuencia, a conductas de aislamiento, incertidumbre y sensación de vulnerabilidad, que pueden afectar el buen desarrollo de las relaciones familiares y generar prácticas parentales negativas que no contribuyen a la educación adecuada de los niños. Algunos autores señalan que, en muchas ocasiones, las reacciones de los padres que viven en pobreza conllevan prácticas punitivas que afectan las relaciones padre-hijo y aumentan la probabilidad de que los niños desarrollen problemas socioemocionales, síntomas psicosomáticos que influyen en su ajuste psicológico (Cabrera \& Guevara, 2007). Así mismo, Mesa y Gómez (2015) resaltan el protagonismo de los aspectos psicoafectivos del profesor en el desarrollo emocional y la salud mental de los niños.

Por esta razón, y a pesar de las diferentes adversidades que enfrentan la familia y los niños en su escuela, los procesos de resiliencia cobran especial relevancia en estos contextos, pues contribuyen de manera significativa al desarrollo educativo de los niños en medio de las situaciones adversas o de vulnerabilidad que puedan enfrentar. En este sentido, la investigación de Crosnoe (2005) sugiere que es necesario hacer intervenciones en la resiliencia desde la educación y las relaciones interpersonales, y propone que el sistema educativo trabaje con propósitos mancomunados con la familia, con el fin de mejorar el bienestar de los niños.

En este orden de ideas, el presente estudio se justifica en la medida en que se pretende analizar las prácticas educativas de padres, madres y profesores que pueden contribuir a generar características resilientes en los niños en medio de contextos de adversidad.

Desde el contexto escolar, Shulman (1992) en su Modelo de Razonamiento Pedagógico explica cuáles deberían ser las prácticas que caracterizan a la buena enseñanza: capacitar a los estudiantes para que disfruten y utilicen sus experiencias de aprendizaje, inculcarles responsabilidades para que lleguen a ser personas solidarias, enseñarles a creer y a respetar a otros y a contribuir al bienestar de la comunidad, ayudarles a desarrollar valores que necesitan para conducirse dentro de una sociedad. Así mismo, los hallazgos de Kagan, Henry, Richardson, Trinkle,
\& LaFrenier (2014), resaltaron la importancia de los programas de bienestar infantil en la escuela, basados en la consolidación de un vínculo de apego secuencial entre el profesor y el niño como factor protector para crecer en resiliencia. De la misma manera, Jennings, Frank, Snowberg, Coccia, \& Greenberg, (2013), resaltan la importancia de que el docente practique el autocontrol de emociones, para promover la resiliencia, el bienestar y la autorregulación en sus alumnos.

En este sentido, la resiliencia ha sido una temática abordada desde hace algunos años como la capacidad de sobreponerse a éstas situaciones adversas o de vulnerabilidad. En esta misma línea, la psicología positiva (Cutuli \& Masten, 2009), ha estudiado la resiliencia haciendo énfasis en la manera de fortalecer los aspectos positivos de los individuos más que en los negativos.Otros estudiosos explican que la resiliencia surge de los diferentes recursos y procesos que moldean una trayectoria de vida positiva. La resiliencia se fundamenta en la interacción entre la persona y el entorno (Cabrera, Aya, \& Cano, 2012; Munist \& cols., 1998; Seligman \& Csikszentmihalyi, 2000), como un conjunto de procesos sociales e intrapsíquicos que hacen posible la salud de un individuo, aun cuando se encuentra en un medio poco saludable. Rodríguez (2006) destaca que los elementos constitutivos de la resiliencia están presentes en todo ser humano y cambian a través del ciclo vital, por lo que promoverla apunta a mejorar la calidad de vida de las personas. Grotberg (2003) la define como "la capacidad del ser humano para hacer frente a las adversidades de la vida, aprender de ellas, superarlas e inclusive, ser transformados por estas" (p.18). En esta misma línea, Luthar, Ciccetti, y Becker, (2008) afirman que implica una adaptación positiva en un contexto de exposición a la adversidad.

Peltonen, Qouta, Diab, \& Punamäki (2014) midieron la resiliencia en niños de acuerdo con el nivel de exposición a un trauma de guerra y a la ausencia o presencia del trastorno de estrés postraumático. En los resultados del grupo de niños resilientes los varones reportaron mayores niveles de resiliencia que las niñas. Richaud (2013), define los niños resilientes como aquellos que resisten la adversidad, saben hacer frente a la incertidumbre y son capaces de recuperarse con éxito de los traumas. Asimismo, sugieren que la formación de la resiliencia debe ser un proceso donde se integre el ambiente familiar, escolar y del vecindario, y a menor edad del niño cuando comienza la intervención, mejores serán los resultados obtenidos.

De acuerdo con lo anterior, es interesante estudiar la manera en la que padres, madres y profesores pueden contribuir al desarrollo de resiliencia en la infancia. Algunos estudios han incluido en su modelo de investigación la influencia de padres y madres, y otros, a los profesores por separado. Sin embargo, no se han encontrado investigaciones que integren el contexto familiar y escolar en un mismo estudio. Esto contribuye a que la alianza entre la familia y escuela se fortalezca y propenda por trabajar mancomunadamente en la formación de la resiliencia en niños.

Para efectos de esta investigación se estudió la resiliencia con base en la propuesta teórica sobre las virtudes humanas de David Isaacs (2003) quien las define como "hábitos operativos buenos" (p.33), es decir, como conductas que se repiten 
una y otra vez, y llegan a formar una característica positiva de la persona. La propuesta de Isaacs sobre las virtudes es reconocida en el contexto familiar y escolar en la educación de los niños. Por tal razón, el presente estudio se fundamenta en sus supuestos teóricos sobre las virtudes como indicadores de resiliencia. El término virtud tiene sus raíces en el griego Arete y el latín virtus. De acuerdo con Aristóteles (1999), se comprende como excelencia añadida a algo como perfección y como una disposición que no es innata, sino resultado del ejercicio de la libertad. Desde la psicología, Cutuli y Masten (2009) definen las virtudes como las características de excelencia y fortaleza de carácter de los seres humanos, que hacen posible que éstos puedan alcanzar su más alto potencial en la vida. Seligman \& Csikszentmihalyi, (2000) se refieren a las fortalezas humanas como indicadores de resiliencia, conceptualizándolas como factores de protección en situaciones de riesgo y como fortalezas que promueven el bienestar y la salud mental en la infancia (Peterson \& Seligman, 2004).

Las virtudes que se contemplaron en este trabajo fueron la generosidad, entendida como entregar lo que se tiene, actuando en favor de otros de forma desinteresada y con alegría aunque implique esfuerzo (Isaacs, 2003). La laboriosidad, la define como el cumplimiento y compromiso en la realización de actividades y tareas. Por su parte, Erikson (2000) se ha referido a la laboriosidad como una fuerza básica que desarrollan los niños aproximadamente entre los 6 y 12 años de edad. Los niños en esta etapa inician su escolaridad, de forma que se enfatiza en el desarrollo de habilidades para llevar a cabo actividades y tareas, así como con el compromiso en la realización de las mismas.

Los padres y profesores, a través de sus prácticas educativas cotidianas, son personas protagonistas en la formación de la resiliencia en los niños. En cuanto a los padres, diversos estudios evidencian que el conflicto marital impacta negativamente el desarrollo de los hijos. Amato y Afifi (2006) reportan que éste se relaciona de forma significativa con la percepción de los hijos de "estar atrapados en medio", que a su vez se asocia con un menor bienestar subjetivo, menor calidad en las relaciones filiales y menor ajuste psicológico (Cabrera \& Guevara, 2007; Schoppe-Sullivan, Schermerhorn, \& Cummings, 2007; Sturge-Apple, Davies, \& Cummings, 2006).

Por su parte, las prácticas parentales posibilitan analizar las diferentes formas de interacción entre padres e hijos, así como la influencia que ejercen en su desarrollo. Las prácticas de los profesores, de igual forma, son determinantes en el desarrollo de los niños. De allí, que los adultos sean ejes significativos en el aprendizaje escolar de los niños, asociado al éxito en la escuela y en la vida misma, al darles pautas de conducta claras y la posibilidad de dar y recibir ayuda eficazmente en situaciones de pérdida y adversidad (Landazábal, Larzelere, \& Owens, 2007).

Para efectos de este estudio se estudiaron las prácticas educativas de padres, madres y profesores. Respecto a ellas, Baumrind (2010) las define como un conjunto de actitudes que son comunicadas a los hijos, las cuales configuran un clima emocional que promueve el bienestar y el desarrollo. Rollins y Thomas (1979) afirman que comprenden intentos de control y de apoyo por parte de los padres hacia los hijos. Henao, Ramírez y Ramírez (2007) exponen que éstas comprenden las preferencias globales de conducta de los padres o figuras de autoridad del niño, inmersas en una relación de bidireccionalidad padres-hijos. Estas prácticas buscan educar a los niños en el contexto familiar y escolar.

Una práctica reconocida es el monitoreo. Stattin y Kerr (2000) y Morrongiello (2005) lo definen como el conocimiento que tienen los padres con relación a sus hijos, resultado del esfuerzo de los padres para conocer lo que hacen sus hijos y a su vez, porque estos últimos comparten información de sus actividades de forma libre y abierta. Alto nivel de monitoreo se establece como factor protector en situaciones adversas de los niños.

El trato rudo se configura como una práctica parental utilizada como estrategia para controlar el comportamiento de los hijos. Frias-Armenta y Mc Closkey (1998) explican que incluye tácticas coercitivas de castigo deliberado. Son prácticas basadas en una disciplina agresiva que implican un bajo nivel de razonamiento y altos niveles de agresividad física, verbal y emocional con los hijos (Sheehan \& Watson, 2008; Roa \& Del Barrio, 2002). Esta práctica se considera un factor de riesgo para los niños.

Rohner (2004) conceptualiza la práctica de la aceptación o apoyo en el marco de formas de relación que son percibidas como cálidas y afectuosas. Por tanto, la calidez se establece como la dimensión de las interacciones que se operacionalizan en conductas físicas, verbales y simbólicas, que usan los padres y/o los profesores, que son percibidas por los niños como expresiones de afecto. La literatura proporciona evidencia de la aceptación o apoyo como un factor de protección parental en diferentes culturas, países y razas y además, y como un indicador de un mejor ajuste en los niños (Khaleque, 2007; Dwairy, 2010). Esta práctica por parte de los profesores se asocia con una actitud positiva en la escuela, el auto-concepto y el rendimiento académico (Gülay, 2011; Conejeros, Gómez-Arizaga, \& Donoso, 2013), así como con baja agresión y altos niveles de auto-regulación y comportamiento prosocial (Merritt, Wanless, Rimm-Kaufman, Cameron \& Peugh, 2012; Baumrind \& cols., 2010).

La afirmación del poderes una práctica parental y escolar que incluye las sanciones que se usan con los niños, entre las que se sitúan el castigo físico, la privación de privilegios, declaraciones coercitivas, rudeza, críticas, expresiones de rabia, órdenes y declaraciones que implican la desaprobación del comportamiento del niño (Smetana,1997). En el ámbito escolar, la afirmación del poder se asocia con comportamientos negativos en los alumnos y bajo rendimiento académico (Henricsson \& Rydell, 2004).

El retiro del afecto se define según Barber y Lovelady (2002) consiste en que los padres manipulan emocionalmente a los niños, así como la ausencia de expresiones de fomento de la autonomía, no hablarles, ignorarlos y tener muestras explícitas de disgusto. Estas se asocian con menores niveles de ajuste psicológico y problemas de comportamiento en los niños.

De acuerdo con lo anterior, se pretende responder las siguientes preguntas de investigación: 
1. ¿Existen diferencias significativas en la resiliencia total y en cada una de las virtudes de acuerdo con el sexo del niño(a)?

2. ¿Existen diferencias significativas en apoyo o aceptación, afirmación del poder y retiro del afecto de acuerdo con el padre, la madre y la profesora del niño?

3. ¿Existen diferencias significativas en el monitoreo y el trato rudo como prácticas educativas de acuerdo con el sexo del padre?

4. ¿Cuál es la asociación entre las prácticas educativas de padres, madres y profesoras y la generosidad, la laboriosidad y la resiliencia total del niño?

5. ¿Qué tanto aportan los factores familiares y escolares en la explicación de la generosidad, la laboriosidad y la resiliencia total del niño?

\section{Método}

\section{Tipo de estudio}

Se trató de un estudio transversal, de análisis correlacional, explicativo.

\section{Participantes}

Se contó con una muestra total de $\mathrm{N}=807$ participantes, es decir, 417 niños con edades entre los 7 y 12 años ( $M=9.87)$ (DS=1.37) provenientes de tres escuelas de zona rural de Cundinamarca y niveles socioeconómicos bajos, nivel 1 y 2. Así mismo, se contó con la participación de 111 mamás, 80 papás y 199 profesoras directoras de grupo. De acuerdo con algunos investigadores que han trabajado con niños, sugieren recoger información adicional de un observador o informante clave que dé cuenta del comportamiento del niño, y que complemente su autorreporte. Pueden ser familiares cercanos, profesores o tutores. La combinación de las respuestas de autorreporte y de los informantes clave puede proveer una medida más válida y confiable (Oros, 2008).

\section{Instrumentos}

Se diseñó un cuestionario sobre las características sociodemográficas de los participantes. Con el propósito de evaluar la resiliencia en la infancia, se tomó la escala de autorreporte tipo Likert Escala de medición de resiliencia, virtudes humanas(Cabrera, Aya-Gómez, \&Guevara-Marín, 2014). Esta escala evalúa 2 dimensiones, generosidad y laboriosidad. Ejemplo de generosidad: "Compartes la comida que te gusta con otros", de laboriosidad: "Sacas tiempo para hacer tus tareas". Escala de 1=nunca, a 4=siempre, a=.76. Se obtuvo un indicador de Resiliencia total con el promedio de la medición de la generosidad y la laboriosidad. Así mismo, con el fin de evaluar los factores familiares y escolares se tuvieron en cuenta los siguientes instrumentos:

Conflicto marital: CPIC-Percepción del niño del conflicto entre padres (Grych, Seid, \& Finchman, 1992), compuesto por nueve preguntas, ejemplo: “¿Mis papás se ponen muy agresivos cuando pelean?". Escala de 1=en desacuerdo a 5=de acuerdo (a=.66- .85).

Apoyo/aceptación: versión reducida del Cuestionario de Aceptación-Rechazo de PARQ, (Rohner, 1991), contiene 15 preguntas sobre los comportamientos de padres y madres que expresan fundamentalmente satisfacción y valoración hacia sus hijos, ejemplo: “¿Mi papá dice cosas buenas sobre mí?”. Se puntúa en valores de 1 a 5 (1=total desacuerdo y $5=$ total acuerdo) (a=.82 -.92).

Monitoreo: Escala sobre conocimiento de los padres de las conductas de los hijos, (Stattin \& Kerr, 2000), se compone de 9 ítems, ejemplo: “¿Mis padres saben quiénes son mis amigos?». Escala de 1 a 5 ( $1=$ no sabe y $5=$ sabe mucho) $(a=.88$ $-.91)$.

Retiro del afecto: cuatro preguntas propuestas por Barber y Lovelady (2002). Se evalúa mediante una escala tipo Likert de 5 puntos ( $1=$ nunca y $5=$ =siempre), ejemplo: "¿Evita mirarme o dirigirme la palabra cuando lo(a) he decepcionado?”. (a=.74-.75).

Trato rudo: Harsh Discipline Scale (Straus, Gelles, \& Steinmetz, 1980) con una escala de 1 a 5 (=nunca, $5=$ =siempre), ejemplo: «Cuando te equivocas, ¿con qué frecuencia tu mamá te pega o te da palmadas?». $(a=.83-.86)$.

Afirmación del poder: Child Rearing Practices Report (CRPR) (Roberts, Block \& Block, 1984), ejemplo: «yo castigo a mi hijo(a) quitándole objetos materiales o privilegios». Escala tipo Likert (1=nunca y $5=$ siempre). a=.82-.87.

Los padres, las madres y los niños contestaron a los cuestionarios de conflicto marital y prácticas educativas. Las profesoras a los de prácticas educativas, a excepción del monitoreo y el trato rudo, con el fin de evitar que contestaran por deseabilidad social.

\section{Procedimiento}

Para la recolección de información, se contactaron tres instituciones educativas de veredas aledañas al municipio de Chía, Cundinamarca. Por medio de cartas dirigidas a los rectores, se explicaron los propósitos del estudio y se solicitó el consentimiento informado de la participación de las familias en el proyecto, los profesores también firmaron su consentimiento. Los cuestionarios fueron aplicados a niños, padres, madres y profesores con ayuda del semillero de investigación. Al final, se ofrecieron conferencias de capacitación a los participantes de acuerdo a los resultados del estudio. 


\section{Resultados}

Los análisis de datos realizados en este estudio se corrieron a través del programa SPSS. Inicialmente se analizó el comportamiento de los datos con el fin de conocer la distribución de los ítems "outliers", es decir aquellos que son inconsistentes con el grueso de la evidencia de datos y reportar los datos perdidos (Tabachnick \& Fidell, 2007).

\section{Análisis preliminares}

En primera instancia se analizaron los estadísticos descriptivos de cada una de las variables del estudio. En la tabla 1 se observa que los papás reportan menores niveles de generosidad en los niños, en contraste, con los otros informantes, a su vez, los niños reportaron mayores niveles de laboriosidad. Las mamás informaron mayores niveles de conflicto, monitoreo, afirmación del poder, retiro del afecto y trato rudo. En cuanto a la escuela, la práctica educativa que más ejercen los profesores es la aceptación o apoyo, en contraste con la afirmación del poder y el retiro del afecto.

\section{Análisis de diferencias de medias en la resiliencia total y en cada una de las virtudes de acuerdo con el sexo del niño(a)}

Al analizar los indicadores de resiliencia por separado, se encontraron diferencias significativas entre niños y niñas en la generosidad $(F(1,397)=6.10 ; p=.01)$ y en la laboriosidad $(F(1,397)=4.42 ; \mathrm{p}<.05)$. Las niñas mostraron niveles más altos de generosidad $(\mathrm{M}=3.05)$ y de laboriosidad $(M=3.56)$ en comparación con los niños (Mgenerosi$\mathrm{dad}=2.90$ y Mlaboriosidad=3.47). En cuanto a la resiliencia total se encontraron también diferencias $(F(1,397)=9.89$; $\mathrm{p}=.00)$. Las niñas mostraron niveles más altos de resiliencia total $(M=3.28)$ en comparación con los niños $(M=3.14)$.

Análisis de diferencias de medias en apoyo o aceptación, afirmación del poder y retiro del afecto de acuerdo con el padre, la madre y la profesora del niño

Las prácticas educativas que se evaluaron en los 3 de educadores del niño fueron la aceptación o apoyo, la afirmación del poder y el retiro del afecto. En los papás, mamás y niños se midieron las 5 prácticas, es decir, también se preguntó por el monitoreo y el trato rudo.

Con el fin de conocer si existían diferencias entre los participantes en su percepción sobre el ejercicio de estas prácticas, se corrieron análisis de diferencias de medias. Se encontraron diferencias significativas entre papá, mamá y profesora en el ejercicio de las prácticas educativas. Concretamente, se encontraron diferencias en la aceptación $(F(2,384)=6.26 ; \mathrm{p}<.01)$, las mamás reportaron niveles más altos (Mmamá=3.58), le siguen los papás (Mpapá=3.53) y luego, las profesoras (Mprofesores=3.41). Al analizar la prueba posthoc, en el análisis de subconjuntos homogéneos no se encontraron diferencias entre papás y mamás ni entre

Tabla 1. Estadísticos descriptivos de las variables del estudio.

\begin{tabular}{|c|c|c|c|c|c|}
\hline Variable & Papás & Mamás & $\begin{array}{c}\text { Percepción hijos } \\
\text { sobre papás }\end{array}$ & $\begin{array}{c}\text { Percepción hijos } \\
\text { sobre mamás }\end{array}$ & Profesoras \\
\hline Conflicto marital & $1.80(.64)$ & $2.19(.57)$ & $2.01(.57)$ & & \\
\hline Monitoreo & $3.30(.61)$ & $3.43(.56)$ & $3.00(.79)$ & $3.34(.59)$ & \\
\hline Apoyo o aceptación & $3.53(.41)$ & $3.50(.30)$ & $3.41(.56)$ & $3.57(.38)$ & $3.38(.40)$ \\
\hline Retiro del afecto & $1.56(.66)$ & $1.74(.71)$ & $1.86(.79)$ & $1.93(.78)$ & $1.60(.65)$ \\
\hline Afirmación del poder & $2.27(.83)$ & $2.52(.80)$ & $2.06(.87)$ & $2.08(.84)$ & $1.84(.75)$ \\
\hline Trato rudo & $1.41(.36)$ & $1.60(.56)$ & $1.62(.65)$ & $1.67(.69)$ & \\
\hline Generosidad & $3.10(.41)$ & $3.02(.43)$ & \multicolumn{2}{|c|}{$2.94(.62)$} & $2.61(.86)$ \\
\hline Laboriosidad & $3.40(.50)$ & $3.41(.43)$ & \multicolumn{2}{|c|}{$3.48(.47)$} & $3.26(.60)$ \\
\hline Resiliencia total & $3.25(.34)$ & $3.21(3.34)$ & \multicolumn{2}{|c|}{$3.21(.44)$} & $2.82(.87)$ \\
\hline
\end{tabular}

Nota: los valores en paréntesis corresponden a las desviaciones estándar. 
papás y profesoras, sin embargo, sí se encontraron entre mamás y profesoras.

En cuanto a la afirmación del poder también se encontraron diferencias significativas $(F(2,374)=33.88$; $p=.000)$. Las mamás reportaron mayores niveles de afirmación del poder hacia sus hijos(as) $(M=2.52)$, le siguen los papás $(\mathrm{M}=2.29)$, y por último, las profesoras $(\mathrm{M}=1.80)$. Los papás y las mamás no presentan diferencias entre ellos al ejercer esta práctica, sin embargo, sí la ejercen más en comparación con las profesoras.

En retiro del afecto no se encontraron diferencias significativas $(F(2,378)=1.74 ; \mathrm{p}=.18)$, sin embargo, las mamás $(\mathrm{M}=1.72)$ reportaron niveles más altos en esta práctica en comparación con las profesores $(M=1.59)$ y los papás $(M=1.57)$.

\section{Análisis de diferencias de medias en el monitoreo y el trato rudo como prácticas educativas de acuerdo con el sexo del padre}

Se encontraron diferencias significativas entre padres y madres en el trato rudo $(F(1,171)=6.68 ; \mathrm{p}=.01)$, más no en el monitoreo $(F(1,177)=.85 ; \mathrm{p}=.36)$. Las madres $(\mathrm{M}=1.60)$ reportaron mayor nivel de trato rudo en comparación con los padres $(\mathrm{M}=1.41)$.

Asociaciones entre las prácticas educativas de padres, madres y profesoras y la generosidad del niño

La generosidad del niño se asoció positiva y significativamente con la aceptación y apoyo del papá $\left(r=.14^{* *}\right)$, de la mamá $\left(r=.13^{* *}\right)$ y el monitoreo del papá $\left(r=.10^{*}\right)$ según reporte del niño, y con la afirmación del poder del profesor $\left(r=-.15^{*}\right)$. Es decir, niveles altos de apoyo y aceptación de ambos padres y el monitoreo del papá se asocian de manera directa con niveles altos de resiliencia en el niño. Por parte del contexto escolar, la afirmación del poder de la profesora se relaciona de manera inversa con la generosidad del niño.

\section{Asociaciones entre las prácticas educativas de padres, madres y profesoras y la laboriosidad del niño}

En cuanto al contexto familiar las prácticas de los padres que se asociaron con la laboriosidad del niño fueron: el monitoreo del papá $\left(r=.39^{* *}\right)$, la aceptación y apoyo de la mamá $\left(r=.31^{* *}\right)$, la aceptación y apoyo del papá $\left(r=.28^{* *}\right)$. Estas dos últimas según la versión del niño. El monitoreo del papá $\left(r=.24^{* *}\right)$ y de la mamá $\left(r=.23^{* *}\right)$ según versión niño. El trato rudo $\left(r=-.10^{*}\right)$ del papá y el conflicto $\left(r=-.18^{* *}\right)$ que percibe el niño de sus padres se relacionaron de forma inversa, es decir, altos niveles del monitoreo del papá, de la mamá y la aceptación de los dos, y bajos niveles de conflicto marital y de trato rudo del papá se asocian con altos niveles de laboriosidad en el niño. Respecto al contexto escolar, la aceptación $\left(r=.25^{\star *}\right)$ y la afirmación del poder $\left(r=-.23^{\star *}\right)$ cor- relacionaron de manera significativa con la laboriosidad, es decir, cuando los profesores aceptan y apoyan a los niños presentan mayores niveles de laboriosidad, y cuando presentan bajos niveles de afirmación del poder los niveles de laboriosidad son altos.

\section{Asociaciones entre las prácticas educativas de padres, madres y profesoras y la resiliencia total}

La resiliencia total correlacionó de manera significativa con: el monitoreo del papá $\left(r=.35^{\star *}\right)$, la aceptación del papá $\left(r=.25^{\star *}\right)$, la aceptación de la mamá $\left(r=.26^{* *}\right)$, el monitoreo del papá $\left(r=.20^{* *}\right)$ y de la mamá $\left(r=.16^{* *}\right)$ según versión niño, es decir, estar pendiente de las actividades de los hijos por parte del papá y de la mamá, aceptarlos y apoyarlos se asocian con altos niveles de resiliencia en los niños.

En cuanto a los factores escolares, la aceptación del docente $\left(r=.19^{*}\right)$ y la afirmación del poder $\left(r=-.19^{*}\right)$ se asociaron de forma significativa con la resiliencia, es decir, altos niveles de apoyo y aceptación de un docente hacia su estudiante y bajos de afirmación del poder, se relacionan con la generación de procesos de resiliencia en niños.

Para la explicación de la generosidad, laboriosidad y resiliencia total del niño, el modelo de regresión lineal múltiple por pasos, se incluyeron las variables que resultaron significativas en los análisis de correlación.

\section{Análisis de la importancia de los factores familiares y escolares en la explicación de la generosidad del niño}

Con el fin de explicar variabilidad de la generosidad del niño, el modelo de regresión lineal por pasos sucesivos compuesto por la percepción del niño del monitoreo del papá, la percepción del niño de la aceptación del papá y de la mamá y la afirmación del poder de la profesora, obtuvo un porcentaje de varianza explicada significativo $\left(R^{2}=.14\right.$; $F(5,77)=2.28 ; p=.05)$. Las variables que explicaron de manera significativa la generosidad fueron en su orden la percepción del niño sobre la aceptación de su papá $(\beta=.44 ; p=.01)$ y la afirmación del poder de la profesora $(\beta=-.21 ; p=.05)$. Es decir, a mayor aceptación del papá y menor afirmación del poder de la profesora más generosidad del niño.

\section{Análisis de la importancia de los factores familiares y escolares en la explicación de la laboriosidad del niño}

Las variables incluidas en este modelo fueron: las familiares: la percepción del niño sobre el conflicto marital, la aceptación y el monitoreo de su papá y de su mamá, el trato rudo del papá y el monitoreo del papá. Dentro de los factores escolares se incluyeron la aceptación y la afirmación del poder de la profesora. Se obtuvo un porcentaje de varianza explicada significativo de la laboriosidad del niño $\left(R^{2}=.16 ; F(5,77)=17.53\right.$; 
$p=.000)$. Las variables que obtuvieron un peso significativo fueron: la percepción del niño de la aceptación de la mamá $(\beta=.25$; $p=.000)$, la percepción del niño sobre el monitoreo del padre $(\beta=.15 ; p=.001)$, el monitoreo del padre sobre el niño $(\beta=.14$; $p=.001)$, la percepción del niño sobre el conflicto entre sus padres $(\beta=-.10 ; p<.05)$, y finalmente, la afirmación del poder de la profesora sobre el niño $(\beta=-.09 ; p<.05)$. Es decir, altos niveles de la aceptación de la mamá, el monitoreo del papá y bajos niveles de conflicto marital y de afirmación del poder de la profesora explican la laboriosidad de los niños.

\section{Análisis de la importancia de los factores familiares y escolares en la explicación de la resiliencia total}

Con el fin de dar cuenta de la variabilidad de la resiliencia total del niño se encontró un aporte significativo de las variables predictoras del modelo $\left(R^{2}=.14 ; F(5,78)=14.88\right.$; $p=.000)$. Las variables que contribuyeron de manera significativa a la explicación de la resiliencia fueron: la aceptación que percibe el niño de su mamá $(\beta=18 ; p=.000)$, la aceptación que percibe el niño de su papá $(\beta=-.09 ; p<.05)$, y el monitoreo que ejerce el papá con el niño $(\beta=.11 ; p<.01)$. Por parte de las profesoras, la aceptación $(\beta=.19 ; p<.05)$ y la afirmación del poder $(\beta=-.19 ; p<.05)$. Es decir, a mayor aceptación percibida por el niño por parte de su mamá, de su papá y el monitoreo que ejerce el papá de las actividades del niño contribuyen al desarrollo de características de resiliencia en los niños, a su vez, la aceptación y apoyo de la docente y bajos niveles de afirmación del poder explican la resiliencia en los niños.

\section{Discusión}

Este estudio tuvo como objetivo contribuir a la explicación de la laboriosidad y la generosidad como indicadores de resiliencia en niños, a partir de ciertas prácticas educativas que ejercen padres y profesores en la familia y en la escuela.

En cuanto a los análisis de la resiliencia según el sexo de los niños, los resultados obtenidos coinciden con los de Cecchet y Thoburn (2014), Weidong, Guangyao, Hua, Ruohong, y Qian (2013), Campbell-Sills, Forde, y Stein, (2009) yReimer (2002), que resaltan que las niñas tienen niveles más altos de resiliencia en comparación con los niños. Este resultado contrasta con lo encontrado por Peltonen, Qouta, Diab, y Punamäki (2014), donde los niños reportaron mayores niveles de resiliencia que las niñas, esta investigación se llevó a cabo teniendo como adversidad el trauma al que los niños estaban expuestos en medio de un contexto de guerra. Esto lleva a pensar, que dependiendo del contexto en el que el niño o la niña se encuentren pueden ser resilientes o no. Ellos pueden serlo en situaciones de guerra y ellas en situaciones que impliquen acciones como compartir o ser sociables con otros.

Según con los hallazgos de Kagan y cols. (2014) que realzan la importancia del profesor en el proceso de la formación de la resiliencia, los resultados de este estudio indicaron que si bien los profesores ejercen las practicas de apoyo y aceptación, y supervisión, éstos lo ejercen en menor proporción que los padres y madres. Este resultado debe llevar a la reflexión de los profesores para que aumenten el ejercicio de las practicas educativas, sobre todo las positivas, y disminuyan las negativas, como la afirmación del poder, con el fin de contribuir a la formación de la resiliencia en los niños(as).

Aunque la práctica que más ejercen los profesores en el contexto educativo es la aceptación y apoyo, se encontraron diferencias en comparación con los papás y las mamás en el contexto familiar. Las mamás son las que más apoyan y aceptan a los hijos, seguidas por los papás (Cabrera, Guevara, \& Barrera, 2006; Cabrera \& Guevara, 2007), y luego las profesoras. Diferencias similares se encontraron en cuanto a la afirmación del poder y el trato rudo, es decir, las mamás son las que más privan de privilegios a los niños y los tratan con rudeza en el proceso educativo.

Cuando el niño percibe altos niveles de monitoreo y aceptación de sus padres se presentan mayores niveles de generosidad y de laboriosidad. Khaleque (2007) y Dwairy (2010) explican que los niños que reciben aceptación y apoyo de sus padres cuentan con un factor que los protege en las relaciones sociales, y esto los lleva a ser generosos y tener más habilidades sociales. Sentirse apoyado y aceptado en la familia y la escuela contribuye a que se sientan más seguros de sí mismos y con alguien en quien confiar.

El apoyo y aceptación del papá y de la mamá, el monitoreo del papá y la afirmación del poder del docente son prácticas educativas que se deben implementar desde el contexto familiar y escolar (LeDoux \& cols. 2002; Gülay, 2011; Merritt \& cols., 2012). Es importante destacar que el apoyo que los papás ofrecen a sus hijos, así como estar pendiente de sus actividades diarias, contribuyen a que el niño sea generoso, así como que el docente le prive de privilegios y le desapruebe algunas conductas (afirmación del poder) se relaciona con la poca presencia de esta característica.

Por su parte, la percepción del niño del conflicto marital entre sus padres se asoció significativamente y de forma negativa con la laboriosidad del niño. Varios estudios han encontrado que los niños que están expuestos al conflicto marital de sus padres demuestran mayores niveles de conductas inadecuadas y de desajuste psicológico (Schoppe-Sullivan, Schermerhorn, \& Cummings, 2007). Estas conductas no se consideran resilientes, puesto que para superar situaciones adversas se asocia con comportamientos positivos o cualidades de la persona. Lo anterior confirma los resultados encontrados en otros estudios sobre resiliencia en niños escolarizados de sectores vulnerables (Villalta \& Saavedra, 2010; Saavedra \& Castro, 2009), en este caso de sector rural y de estratos bajos con pocos recursos económicos, y además, indica un terreno fértil para que los profesores puedan fortalecer y promover patrones innovadores de interacción didáctica que lleven a generar características de resiliencia en sus educandos.

Colombia es un aún un país de contrastes. Por un lado, existe población infantil que cuenta con grandes recursos económicos y facilidades de acceso a la privilegios educativos y sociales, no obstante, por otro lado, los niños colombianos 
adolecen de carencias y recursos que podrían enfrentarse con la educación de habilidades y virtudes propias de personas resilientes con el fin de hacer frente a las situaciones adversas de este país en proceso de desarrollo.

En este orden de ideas, es muy probable que padres y profesores cuenten con mayores recursos internos personales, en comparación con los niños que se encuentran en procesos de formación y adquisición de habilidades resilientes, y a su vez, que pertenecen a sectores sociales como el rural y estratos socioeconómicos bajos. Sin embargo, a pesar de estar inmersos en estas condiciones desfavorables, los niños cuentan con estas figuras educativas y significativas que deben propender por ser modelo de trabajo de la laboriosidad y la generosidad en sus vidas, así como ejercer prácticas educativas que los lleven a adquirirlas. Como lo expone Richaud (2013) los niños que pueden llegar a desarrollar características de resiliencia son los que enfrentan situaciones adversas, asumen los retos inciertos de la vida y demuestran la capacidad de salir exitosos en los traumas propios del contexto. El ambiente familiar y escolar son los principales contextos que fomentan la resiliencia en los niños.

Los profesores y los padres como agentes protagónicos del proceso educativo de los niños pueden generar espacios en el contexto escolar y familiar para generar procesos de resiliencia. Esto se pude lograr, en palabras de García (2013), mediante la enseñanza de comportamientos y aprendizajes que posibiliten la mejora y el enriquecimiento personal de los educandos. Por eso, el modo más adecuado para evaluar la calidad del proceso educativo, es atender a los resultados reales de aprendizaje intelectual y moral que promueve en los niños los comportamientos resilientes. Esto coincide con lo expuesto por Shulman, (1992), quien explica que es importante propender el fomento de responsabilidades en los niños (laboriosidad) y actitudes solidarias y de ayuda a los demás (generosidad) que los lleven a pensar en otros, y como consecuencia, aprender a enfrentar situaciones adversas desde el proceso educativo.

En este sentido, Green, Walker, Hoover-Dempsey, y Sandler (2007), plantean que el profesorado que posee una actitud positiva respecto a la participación de las familias en las escuelas obtiene importantes beneficios, pues logra conocer a las familias de sus alumnos, facilitando no solo la participación sino también el desarrollo más fluido y estrecho de la relación, para fomentar procesos de resiliencia.

\section{Conclusiones y consideraciones finales}

En síntesis, para promover la generosidad y la laboriosidad como indicadores de resiliencia en la infancia, en la familia es importante buscar que los padres y madres ejerzan la aceptación y el apoyo a los niños y estar pendiente de sus actividades, así como, en la escuela la aceptación y el apoyo del profesor y la poca afirmación del poder. Son factores preponderantes a la hora de enfrentar situaciones de adversidad en la infancia.
Según los resultados de esta investigación, si bien los padres y las madres son considerados figuras significativas al interior de la familia, el profesor es un factor del que no se puede prescindir cuando se quiere promover el desarrollo de la resiliencia en los niños. Ellos juegan un papel significativo, sobre todo en el contexto de algunos niños de estratos bajos y de condiciones rurales que crecen solos, a razón de que sus padres deben trabajar bastantes horas al día y permanecer fuera de casa. En contraste, en necesario reflexionar que en Colombia no se le otorga el suficiente reconocimiento y prestigio al profesor, por parte de los padres y a la inversa, y más que una separación de funciones, es menester establecer una relación integradora para que juntos y hacia un mismo fin, busquen el beneficio del niño. Tanto los padres, las madres como los profesores pueden establecer una relación positiva, en cuanto se convierte en una relación reparadora, pero también negativa en la medida que no se ejercen las prácticas educativas indicadas.

Los resultados obtenidos permiten aportar elementos relevantes para el diseño de programas orientados a fomentar desde el contexto familiar y escolar la resiliencia en niños, y de acuerdo con Richaud (2013) cuando la intervención comienza a menoredad del niño, mejores serán los resultados obtenidos. Sin embargo, para el desarrollo de investigaciones futuras se deben contemplar otros contextos, como las zonas urbanas, la influencia de los pares, examinar los diferentes niveles socioeconómicos, así como otras situaciones adversas, por ejemplo, contar con un solo padre o madre por separación o divorcio o la muerte de uno de ellos al interior de la familia, mostrar bajo rendimiento académico, o experimentar matoneo o intimidación en la escuela. Así mismo, podría profundizarse en otros aspectos como el orden de nacimiento del niño, la influencia de la familia extensa, la participación de otros profesores diferentes a los directores de grupo o los amigos.

Llevar a cabo estudios transculturales podrían explicar los factores que contribuyen a explicar estas características en diferentes países.

\section{Referencias}

Amato, P. R. \& Afifi, T. D. (2006). Feeling caught between parents: Adult children's relations with parents and subjective well-being. Journal of Marriage and Family, 68, 222-235.

Aristóteles (1999). Ética Nicomaquea. México: Editorial Porrúa.

Barber, B. K. \& Lovelady, E. (2002). Violating the self: parental psychological control of children and adolescents. Em B. K. Barber (Org.), Intrusive Parenting. How psychological control affects children and adolescents. American Psychological Association, Washington.

Baumrind, D., Larzelere, R. E., \& Owens, E. (2010). Effects of preschool parents' power assertive patterns and practices on adolescent development. Parenting: Science and Practice, 10, 157-201. 
Cabrera, V., Guevara, I., \& Barrera, F. (2006). Relaciones maritales, relaciones paternas y su influencia en el ajuste psicológico de los hijos. Acta Colombiana de Psicología, 9(2), 115-126.

Cabrera, V. \& Guevara, I. (2007). Relaciones familiares y ajuste psicológico: dos estudios en adolescentes de familias colombianas, Bogotá, Colombia: Ediciones Uniandes.

Cabrera-García, V. E., Aya-Gómez, V. L., \& Guevara-Marín, I. P. (2014). Diseño y análisis psicométrico de una escala de resiliencia en infantes. Revista Facultad de Medicina, 62(2), 213-220.

Cabrera, V. E., Aya, V. L.,\& Cano, A. M. (2012). Una propuesta antropológica para la comprensión de la resiliencia en niños: las virtudes humanas. Persona y Bioética, 16(2), 149-164.

Campbell-Sills, L., Forde D.R., \& Stein, M. B. (2009). Demographic and childhood environmental predictors of resilience in a community sample. Journal of Psychiatric Res, 43(12), 1007-1012.

Cecchet, S. \& Thoburn, J. (2014). The Psychological Experience of Child and Adolescent Sex Trafficking in the United States: Trauma and Resilience in Survivors. Psychological Trauma. Theory, Research, Practiceand Policy, 6(5), 482-493.

Conejeros-Solar, M. L., Gómez-Arizaga, M. P., \& Donoso-Osorio, E. (2013). Perfil docente para alumnos/as con altas capacidades. Magis, Revista Internacional de Investigación en Educación, 5(11), 393-411.

Crosnoe, R. (2005). Double Disadvantage or Signs of Resilience?The Elementary School Contexts of Children from Mexican Immigrant Families.American Educational Research Journal, 42(2),269-303.

Cutuli, A. S. \& Masten. J. J. (2009). The Encychopedia of Positive Psychology (Edited by Shane J. López, the Clifton Strenghts Institute and Gallup). United Kingdom: A John Wiley \& Sons, Ltd. Publication

Dwairy, M. (2010). Parental acceptance-rejection: A fourth crosscultural research on parenting and psychological adjustment of children. Journal of Child and Family Studies, 19(1), 30-35

Erikson, E. (2000). El ciclo vital completado. Barcelona: Paidós.

Fonagy, P., Steele, M., Steele, H., Higgitt, A., \& Target, M. (1994).The Emmanuel Miller Memorial Lecture 1992. The theory and practice of resilience. Journal of Child Psychology and Psychiatry and Allied Disciplines, 35, 231-257.

Frias-Armenta, M. \& McCloskey, L. A., (1998). Determinants of harsh parenting in Mexico.Journal of Abnormal Child Psychology, 26, 129-139.

García, M. (2013). En el aula y fuera de ella: educar con la vida. Edetania: estudios y propuestas socio-educativas, 43,83-95.
Green, C., Walker, J., Hoover-Dempsey, K., \& Sandler, H. (2007). Parents' Motivations for Involvement in Children's Education: An Empirical Test of a Theoretical Model of Parental Involvement. Journal of Educational Psychology, 99 (3), 532-544.

Grotberg, E. (2003). La resiliencia en el mundo de hoy.España: Gedisa.

Grych, J. H., Seid, M., \& Finchman, F. (1992).Assessing marital conflict from the child's perspective: The chidren's percepction of interparental conflict scale. Child Development, 63, 558-572.

Gülay, H. (2011). Assessment of the prosocial behaviors of young children with regard to social development, social skills, parental acceptance-rejection and peer relationships. Journal of Instructional Psychology, 38(3),164-172.

Henao, G., Ramírez, C., \& Ramírez, L. (2007).Las prácticas educativas familiares como facilitadores del proceso de desarrollo en el niño y niña. Revista Ágora Universidad de San Buenaventura, 7(2). p. 235-237.

Henricsson, L., \& Rydell, A. (2004). Elementary school children with behavior problems: Teacher-child relations and self-perception. A prospective study. Merrill - Palmer Quarterly, 50(2), 111-138.

Isaacs, D. (2003). La educación de las virtudes humanas y su evaluación. Eunsa, España.

Jennings, P., Frank, J., Snowberg, K., Coccia, M., \& Greenberg, M. (2013). Improving Classroom Learning Environments by Cultivating Awareness and Resilience in Education (CARE): Results of a Randomized Controlled Trial. School Psychology Quarterly, 28(4), 374-390.

Kagan, R., Henry, J., Richardson, M., Trinkle, J., LaFrenier, A. (2014). Evaluation of Real Life Heroes Treatment for Children with Complex PTSD. Psychological Trauma: Theory, Research, Practice, and Policy, 6(5), 588-596.

Khaleque, A. (2007). Parental acceptance-rejection theory: Beyond parent-child relationship. International Society for interpersonal Acceptance and Rejection, 1(1), 2-4.

Landazábal, D., Cardona, M., Espinosa, L., Garzón, B., Jiménez, B., González, L., \& Rodríguez, N. (2007). Una luz que brilla: La resiliencia. Análisis de investigaciones realizadas en la última década. Colombia: Universidad Nacional Abierta y a Distancia UNAD.

LeDoux, S., Miller, P., Choquet, M., \& Plant, M. (2002). Family structure, parent-child relationships, and alcohol and other drug use among teenagers in France and the United Kingdom. Alcohol \& Alcoholism, 37, 52-60.

Luthar S, Ciccetti D., \& Becker, B. (2008). The construct of resilience: A critical evaluation and guidelines for future work. Child Development, 71(3), 543-562. 
Meresman, S. (2005). Escuelas Promotoras de Salud, Curso Internacional de Promoción de la salud CIP/ Organización Panamericana de la salud 2005. Argentina: Facultad Latinoamericana de Ciencias Sociales.

Merritt, E. G., Wanless, S. B., Rimm-Kaufman, S., Cameron, C., \& Peugh, J. L. (2012). The contribution of teachers' emotional support to children's social behaviors and self-regulatory skills in first grade. School Psychology Review, 41(2), 41-159.

Mesa, A. \& Gómez, A. (2015). Salud Mental, Función Docente y Mentalización en la Educación Preescolar. Psicologia Escolar e Educacional, 19(1), 117-125.

Morrongiello, B.A. (2005). Caregiver supervision and child-injury risk: I. Issues in defining and measuring supervision; II. Findings and directions for future research.Journal of Pediatric Psychology, 30(7), 536-552.

Munist, M., Santos, H., Kotliarenco, M., Suárez, E., Infante, F., \& Grotberg, E. (1998). Manual de identificación y promoción de la resiliencia en niños y adolescentes. Organización Panamericana de la salud.

Oros, L. B. (2008). Avances metodológicos en evaluación de emociones positivas en niños en riesgo social. Evaluar, Laboratorio de evaluación psicológica y educativa, 8, 20-33.

Peltonen, K., Qouta, S., Diab, M., \& Punamäki, R. (2014). Resilience among children in war: The role of multilevel social factors. Traumatology, 20(4), 232-240.

Peterson, C. \& Seligman, M. (2004). Character strengths and virtues: a handbook and classification. Oxford University Press.

Reimer, M. S. (2002). Gender, risk, and resilience in the middle school context. Children \& Schools, 24(1), 35-47.

Richaud, M. C. (2013). Contributions to the study and promotion of resilience in socially vulnerable children. American Psychologist, 68 (8), 751-758.

Roa, L. \& Del Barrio, M. V. (2002). Cuestionario de percepción de crianza para niños y adolescentes. Psicología Educativa, 8(1), 3751.

Roberts, G. C., Block, J. H., \& Block, J. (1984). Continuity and change in parents' child-rearing practices. Child Development, 55, 586 -597 .

Rodríguez, M. S. (2006). Resiliencia: otra manera de ver la adversidad. Ed. Pontificia Universidad Javeriana Facultad de Teologia. Bogotá, Colombia.

Rohner, R. P. (1991). Handbook for the study of parental acceptance and rejection. University of Connecticut: Center for the study of parental acceptance and rejection.
Rohner, R. P. (2004). The parental acceptance-rejection syndrome: Universal correlates of perceived rejection. American Psychologist, $59,830-840$.

Rollins, B. C. \& Thomas, D. L. (1979).Parental support, power and control techniques in the socialization of children. Em E. R. Burr \& cols. (Orgs.), Contemporary theories about the family. New York, Free Press; pp. 317-364.

Rutter M. (2006). Implications of resilience concepts for scientific understanding. Em Annals New York Academy of Sciences, 1-12.

Saavedra, E. \& Castro, A. (2009). Escala de Resiliencia Escolar. Santiago, Chile: CEANIM.

Schoppe-Sullivan, S., Schermerhorn, A. C., \& Cummings, E. M. (2007). Marital conflict and children's adjustment: Evaluation of the parenting process model. Journal of Marriage and Family, 69(5), 1118-1134.

Seligman, M. E. P. \& Csikszentmihalyi, M. (2000). Positive Psychology: An Introduction. American Psychologist, 55(1), 5-14.

Sheehan, M. \& Watson, M. (2008). Reciprocal Influences between Maternal Discipline Techniques and Aggression in Children and Adolescents. Aggressive Behavior, 34, 245- 255.

Shulman, L. (1992). Ways of seeing, ways of knowing, ways of teaching, ways of learning about teaching. Journal of Curriculum Studies, 1, 393-396.

Smetana, J. G. (1997). Parenting and the development of social knowledge reconceptualized: A social domain analysis. Em J. E. Grusec \& L. Kuczynski (Orgs.), Parenting and the Internalization of Values(pp. 162-192). New York: Wiley.

Stattin, H. \& Kerr, M. (2000). Parental monitoring: a reinterpretation. Child development, 71, 1072-1085.

Straus, M. A., Gelles, R. J., \& Steinmetz, S. K. (1980). Behind closed doors: Violence in the american family. Beverly Hills, CA: Sage.

Sturge-Apple, M. L., Davies, P.T., \& Cummings, E.M. (2006). Hostility and withdrawal in marital conflict: Effects on parental emotional unavailability and inconsistent discipline. Journal of Family Psychology, 20, 227-238.

Tabachnick, B. G. \& Fidell, L.S. (2007). Using multivariate statistics (5th ed.) Boston: Allyn and Bacon.

Villalta, M. A. \& Saavedra, E. (2010). Cultura escolar, prácticas de enseñanza y resiliencia en alumnos y profesores de contextos sociales vulnerables. Universitas Psychologica, 11(1), 67-78.

Weidong, J., Guangyao, L., Hua, T. Ruohong, C. \& Qian, Y. (2013). Relationship between resilience and social support, coping style of children in middle school.Em European Psychiatry, Abstracts of the 21th European Congress of Psychiatry, 28, Supplement 1. 
Recebido em: 27/08/2015

Reformulado em: 25/07/2016

Aprovado em: 18/08/2016

\section{Sobre os autores}

Victoria Eugenia Cabrera García (victoria.cabrera@unisabana.edu.co)

Maestría En Psicología de Investigación Psicosocial - Universidad de Los Andes Colombia. Especialización En Educación y Asesoría Familiar Universidad de La Sabana. Psicología - Universidad de La Sabana

Viviana Lucía Aya Gómez (viviana.aya@unisabana.edu.co)

Maestría En Psicología - Universidad de La Sabana. Especialización En Desarrollo Personal y Familiar - Universidad de La Sabana. Psicología Universidad de La Sabana

Diana Sthefanía Muñoz Gómez (diana.munoz5@unisabana.edu.co)

Especialización En Desarrollo Personal y Familiar - Universidad de La Sabana Derecho - Universidad de La Sabana

Ivón Paola Guevara Marín (ivon.guevara@unisabana.edu.co)

Maestría En Psicología - Universidad de Los Andes Colombia. Especialización En Desarrollo Personal y Familiar - Universidad de La Sabana. Psicología - Universidad de La Sabana

Andrés Mauricio Cano Rodas (andres.cano@unisabana.edu.co)

Maestría en Trabajo Social con énfasis en Familia y Redes Sociales - Universidad Nacional de Colombia. Universidad de La Sabana. Especialización En Desarrollo Personal y Familiar - Universidad de La Sabana. Ingeniería Civil - Universidad Nacional de Colombia. 